Original Article

\title{
ANTIMICROBIAL PROPERTIES OF SEVEN BROWN ALGAE HARVESTED FROM THE COAST OF SIDI BOUZID (EL JADIDA-MOROCCO)
}

\author{
NAZHA SAMRI, LAILA HSAINE, SOUKAINA ELKAFHI, SAMIRA KHLIFI, SAMIRA ETAHIRI
}

Marine Biotechnology and the Environment Laboratory (BIOMARE) Faculty of Science, University Chouaib Doukkali-El Jadida-Morocco Email: samri.nazha@gmail.com

Received: 12 Aug 2020, Revised and Accepted: 31 Oct 2020

\begin{abstract}
Objective: This work aims at the screening of the antimicrobial activity of the seven brown marine algae of theCoast of Sidi Bouzid (El JadidaMorocco).

Methods: The aim of this study was to evaluate the antimicrobial activity of seven brown marine algae against three Gram-positive bacteria (Staphylococcus epidermidis, Staphylococcus aureus and Streptococcus pyogenes). Three Gram-negative bacteria (Escherichia coli, Pseudomonas aeruginosa and Klebsiella pneumonia) and two fungi (Aspergillus Niger and Candida tropicalis). Thus, 35 algal extracts were prepared with five organic solvents methanol/water, methanol, dichloromethane/methanol, dichloromethane and ethyl acetate. The antibacterial activity was evaluated through the disk diffusion method.
\end{abstract}

Results: Data revealed that the Staphylococcus aureus bacteria was the most sensitive pathogen by showing the highest zone of inhibitions of 20 $\mathrm{mm}$ with the lowest Minimum Inhibitory Concentration (MIC) of $2 \mu \mathrm{gmL}^{1}$ methanol/water extract of Cystoseira tamariscifolia. Whereas, antifungal activity, the highest zone of inhibitions of $21 \mathrm{~mm}$ and $22 \mathrm{~mm}$ with the lowest Minimum Inhibitory Concentration (MIC) of $5 \mu \mathrm{gmL}{ }^{-1}$ was respectively shown in the methanol/water extract of Laminaria ochroleuca against Candida tropicalis and in the dichloromethanolic extract of Sargassum vulgare against Aspergillus niger.

Conclusion: The results indicate that these algal extracts can further be analyzed and purified for relevant antibacterial and antifungal compounds which can be used in therapeutics and other applications.

Keywords: Brown algae, Antibacterial activity, Antifungal activity, Solvent extracts

(C) 2020 The Authors. Published by Innovare Academic Sciences Pvt Ltd. This is an open access article under the CC BY license (http://creativecommons.org/licenses/by/4.0/) DOI: http://dx.doi.org/10.22159/ijpps.2020v12i12.39362. Journal homepage: https://innovareacademics.in/journals/index.php/ijpps.

\section{INTRODUCTION}

Marine species, comprising approximately a half of the total global biodiversity, are a rich source of structurally diverse bioactive compounds with various biological activities. Thus, their importance as a source of novel bioactive substances is growing rapidly. Among marine organisms, algae are rich sources of bioactive compounds with various biological activities. Recently, their value as a source of novel bioactive substances has become important. Moreover, researchers have revealed that marine algal originated compounds exhibit various biological activitie [1-3].

Most of the secondary metabolites biosynthesized by the marine algae are well-known for their cytotoxic [4], anti-inflammatory [5-8] property, their numerous studies have revealed the anti-bacterial [9$13]$, antifungal activity $[14,2,3]$ and antioxidant $[15,16]$ properties in different macro-algae.

The growing resistance of bacteria to present antibiotics is a major problem worldwide. One way to prevent this resistance is the development of new compounds different from the existing synthetic antimicrobials. Thus, the search for new natural sources of marine ecosystems has led to the isolation of new algal antibiotics such as acrylic acid, halogenated aliphatic compounds, terpenes, sulfurcontaining heterocyclic compounds and phenolic inhibitors [17-19].

This work aims to evaluate the antimicrobial activity of seven seaweeds collected from the coast of Sidi Bouzid, El Jadida against clinical multidrug resistant bacteria and fungi in order to discover new compounds with important antimicrobial activity.

\section{MATERIALS AND METHODS}

\section{Sampling}

The brown algae were carefully removed manually along the coast of

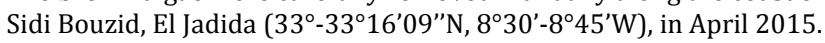

The collected algae were: Sargassum muticum (Yendo) Fensholt 1955, Fucus spiralis Linnaeus 1753, Cystoseira tamariscifolia (Hudson) Papenfuss 1950, Sargassum vulgare C. Agardh 1820, Cystoseira humilis var. myriophylloides (Sauvageau) J. H. Price and D. M. John 1978, Bifurcaria bifurcata R. Ross 1958 and Laminaria ochroleuca Bachelot Pylaie 1824. Algae were initially washed in seawater to remove the macroscopic epiphytes, particles and other extraneous matters and then rinsed in distilled water. Later, algae were air-dried at room temperature and ground to a fine powder for further analysis.

Preparation of extracts

The prepared powder for each species was extracted in different solvent: methanol/water (40/60), methanol, dichloromethane/ methanol $(50 / 50)$, dichloromethane and ethyl acetate at a rate of $1 \mathrm{~g}$ of alga powder/5 $\mathrm{ml}$ of solvent during $72 \mathrm{~h}$ at ambient temperature according to the extraction protocol described by caccamese [20], then the extracts are filtered on Whatman paper $\mathrm{N}^{\circ} 1$ and evaporated in a rotary evaporator. The dry extracts obtained are stored at $4{ }^{\circ} \mathrm{C}$. For methanol water extract the evaporated extract was also lyophilised until later use for the biological tests.

\section{Bacterial and fungal pathogens}

The strains used for these test were obtained from the Collection of Institute Pasteur of Paris (CIP), from American Type Culture Collection (ATCC) and from Mohamed V Hospital (El Jadida Morocco). The Gram-positive bacteria included: Staphylococcus epidermidis, Staphylococcus aureus (ATCC25925) and Streptococcus pyogenes. Gram-negative bacteria were Pseudomonas aeruginosa (ATCC9027), Escherichia coli (ATCC10536) and Klepseilla pneumonia. Fungi used were Candida tropicalis (ATCC127581) and Aspergillus niger (CIP 1275). 


\section{Antimicrobial bioassays}

Antibacterial assays were carried out using the agar disk-diffusion assay Bauer [21]. Three colonies of each bacterium were removed with a wire loop from the original culture plate, and were introduced into a test tube containing $5 \mathrm{ml}$ broth. An overnight culture yielded a suspension of $10^{6}$ bacteria/ml (evaluated by the absorbance value of 0.5 at $620 \mathrm{~nm}$ ) with sterile water to inoculate Petri dishes containing culture media (12 ml Mueller-Hinton agar, $3 \mathrm{~mm}$ thick). Plates were dried for $30 \mathrm{~min}$ before inoculation. The organic extracts were tested using paper disks (6 $\mathrm{mm}$ diameter) impregnated with the $150 \mu \mathrm{g}$ of each extract, after the temperature was equalized at $4{ }^{\circ} \mathrm{C}$; the plates were incubated overnight at $37^{\circ} \mathrm{C}$. Diameters of inhibitory zones were then measured. The Streptomycin $(100 \mu \mathrm{g})$ and Ofloxacine $(50 \mu \mathrm{g})$ susceptibility test discs were used as the positive control.

For fungicidal activity, zones of inhibition were determined after 24 $\mathrm{h}$ of incubation at $27^{\circ} \mathrm{C}$. Discs impregnated with standard antibiotics such as Amphotericin B were used at $100 \mu \mathrm{g}$ as reference in the test of antifungal activity. In addition, control disks were prepared with each solvent. All tests were at least triplicate.

The antimicrobial activity was classified from low active (+: diameter of inhibition $<10 \mathrm{~mm})$, moderately active $(++: 10 \mathrm{~mm} \leq$ diameter of inhibition $<15 \mathrm{~mm}$ ) to highly active $(+++: 15 \mathrm{~mm} \leq$ diameter of inhibition) and inactive (-: no/very hazy inhibition zone) [22].

\section{Minimum inhibitory concentration (MIC)}

The minimum inhibitory concentration (MIC), using microdilution plate method with resazurin Sarker [23], was determined. Briefly, the 96-wellmicroplate was prepared by dispensing $100 \mu \mathrm{l}$ of Mueller-Hinton broth (bacteria strain) or PDA (fungi strains) into each well. $100 \mu \mathrm{l}$ from the stock solution of tested extract (concentration of $40 \mathrm{mg} / \mathrm{ml}$ ) were added into the first row of the plate. Then, two-fold, serial dilutions were performed by transferring $100 \mu \mathrm{l}$ of solution from one row to another, using a multichannel pipette. The obtained concentration range was from 20 $\mathrm{mg} / \mathrm{ml}$ to $3.10-4 \mathrm{mg} / \mathrm{ml}$. $10 \mu \mathrm{l}$ of each $106 \mathrm{CFU} / \mathrm{ml}$ bacterial suspension were added to wells. Finally, $10 \mu \mathrm{l}$ of resazurin solution were added. Resazurin is an oxidation-reduction indicator used for the evaluation of microbial growth. It is a blue non-fluorescent dye that becomes pink and fluorescent when reduced to resorufin by oxidoreductases within viable cells. The inoculated plates were incubated at $37{ }^{\circ} \mathrm{C}$ for $24 \mathrm{~h}$ for bacteria strains but for fungi strains the plates were incubated at $22{ }^{\circ} \mathrm{C}$ for $48 \mathrm{~h}$. MIC was defined as the lowest concentration of the tested algae extracts that prevented resazurin color change from blue to pink. Antibiotic streptomycin and ofloxacin, dissolved in Mueller-Hinton broth, were used as positive controls for bacteria strains, for fungi strains amphotericin $B$ was used as antibiotic. Solvent control test was performed to study an effect of $10 \%$ DMSO on the growth of bacteria. It was observed that 10\% DMSO did not inhibit the growth of bacteria. Each test included growth control and sterility control. All tests were performed in duplicate and MICs were constant.

\section{RESULTS AND DISCUSSION}

The paper described the antimicrobial activity of seven brown algae collected from the coast of Sidi Bouzid El Jadida against clinical multidrug resistant bacteria and fungi. The results of the screening of antibacterial and antifungal activities against bacteria and yeast are summarized in tables 1 and 2 .

\section{Antibacterial activity}

The antibacterial activity of thirty five organic extracts (methanol/water (40/60), methanol, dichloromethane/methanol (50/50), dichloromethane and ethyl acetate), obtained from seven seaweeds species against six pathogenic bacteria was studied in

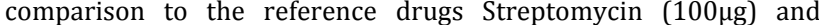
Ofloxacine $(50 \mu \mathrm{g})$. Results obtained were reassembled in table 1.

Table 1: Antibacterial activity of seven brown seaweeds extracts against pathogenic bacteria

\begin{tabular}{|c|c|c|c|c|c|c|c|}
\hline & & Diameter of in & ition (mm) & & & & \\
\hline & & Gram+bacteria & & & Gram-bacteria & & \\
\hline Algae & $\begin{array}{l}\text { Solvent } \\
\text { extraction }\end{array}$ & $\begin{array}{l}\text { Streptococcus } \\
\text { pyogenes }\end{array}$ & $\begin{array}{l}\text { Staphylococcus } \\
\text { aureus }\end{array}$ & $\begin{array}{l}\text { Staphylococcu } \\
\text { s epidermidis }\end{array}$ & $\begin{array}{l}\text { Klepsiella } \\
\text { pneumoniae }\end{array}$ & $\begin{array}{l}\text { Pseudomonas } \\
\text { aeruginosa }\end{array}$ & $\begin{array}{l}\text { Escherichi } \\
\text { a Coli }\end{array}$ \\
\hline & MW & $7 \pm 0.461$ & $18 \pm 0,763$ & $11 \pm 0.768$ & $9 \pm 0.721$ & $12 \pm 0.577$ & - \\
\hline & $\mathrm{MeOH}$ & $9 \pm 0.881$ & $9 \pm 0,440$ & $13 \pm 0.577$ & $7 \pm 0.000$ & - & $14 \pm 0.408$ \\
\hline S. muticum & $\mathrm{MeOH} / \mathrm{DC}$ & - & - & $9 \pm 1.424$ & - & $7 \pm 0.000$ & - \\
\hline & DC & - & $15 \pm 0.5$ & - & $9 \pm 0.577$ & $7 \pm 0.577$ & $8 \pm 0.816$ \\
\hline & EA & - & $11 \pm 0.927$ & $10 \pm 0.000$ & $8 \pm 1.000$ & $13 \pm 0.712$ & \\
\hline & MW & $9 \pm 0.881$ & $19 \pm 0.577$ & $15 \pm 0.433$ & - & $19 \pm 0.577$ & - \\
\hline & $\mathrm{MeOH}$ & $13 \pm 1.154$ & $8 \pm 1.00$ & $15 \pm 0.635$ & $16 \pm 0.950$ & - & $8 \pm 1.224$ \\
\hline F. spiralis & $\mathrm{MeOH} / \mathrm{DC}$ & $7 \pm 0.333$ & - & $8 \pm 0.416$ & - & $10 \pm 1.527$ & $12 \pm 0.734$ \\
\hline & DC & - & $10 \pm 0.850$ & - & - & $10 \pm 0,000$ & - \\
\hline & EA & - & 10 & $10 \pm 0.548$ & - & $12 \pm 1.154$ & - \\
\hline & MW & $10 \pm 1.527$ & $20 \pm 0.731$ & $15 \pm 0.895$ & $7 \pm 0.000$ & $15 \pm 0.925$ & - \\
\hline & $\mathrm{MeOH}$ & $7 \pm 0.440$ & $7 \pm$ & $12 \pm 0.635$ & $7 \pm 0.000$ & $8 \pm 0.000$ & $7 \pm 0.000$ \\
\hline C. tamariscifolia & $\mathrm{MeOH} / \mathrm{DC}$ & $7 \pm 0.881$ & - & $8 \pm 0.000$ & - & - & - \\
\hline & DC & $7 \pm 0.577$ & $10 \pm$ & $7 \pm 1.000$ & - & - & - \\
\hline & EA & $10 \pm 1.000$ & $10 \pm$ & $17 \pm 0.333$ & - & $10 \pm 1.154$ & - \\
\hline & MW & $8 \pm 0.666$ & $18 \pm 0.440$ & - & $9 \pm 0.577$ & $19 \pm 0.440$ & - \\
\hline & $\mathrm{MeOH}$ & $7 \pm 0.440$ & $7 \pm 0.000$ & $12 \pm 0.907$ & $9 \pm 1.527$ & $7 \pm 0.333$ & $7 \pm 0.577$ \\
\hline S. vulgare & $\mathrm{MeOH} / \mathrm{DC}$ & - & - & $10 \pm 1.617$ & - & $7 \pm 0.000$ & - \\
\hline & DC & $9 \pm 1.00$ & $9 \pm 0.392$ & - & - & - & - \\
\hline & EA & $10 \pm 0.577$ & $10 \pm 0.000$ & $10 \pm 1.386$ & $9 \pm 0.577$ & - & - \\
\hline & MW & - & $10 \pm 1.404$ & $9 \pm 0.000$ & $8 \pm 1.047$ & - & $9 \pm 0.577$ \\
\hline & $\mathrm{MeOH}$ & $11 \pm 1.763$ & $10 \pm 0.721$ & $10 \pm 1.732$ & $9 \pm 0.000$ & $10 \pm 0,866$ & - \\
\hline C. humilis & $\mathrm{MeOH} / \mathrm{DC}$ & - & - & - & $8 \pm 0.503$ & - & - \\
\hline & DC & $10 \pm 1.424$ & $12 \pm 1.747$ & - & - & - & $8 \pm 0.000$ \\
\hline & EA & & $10 \pm 0.000$ & $17 \pm 0.577$ & $9 \pm 1.154$ & - & $8 \pm 0.000$ \\
\hline & MW & $7 \pm 0.333$ & $12 \pm 1.069$ & $15 \pm 0.881$ & $10 \pm 0.000$ & - & $9 \pm 1.201$ \\
\hline & $\mathrm{MeOH}$ & $7 \pm 0.881$ & $10 \pm 1.550$ & $10 \pm 0.000$ & - & $8 \pm 1.154$ & $7 \pm 0.726$ \\
\hline L. digitata & $\mathrm{MeOH} / \mathrm{DC}$ & $8 \pm 0.577$ & - & - & - & $8 \pm 1.201$ & - \\
\hline & DC & $9 \pm 0.000$ & - & - & - & $8 \pm 1.154$ & - \\
\hline & EA & - & $10 \pm 0.466$ & - & $8 \pm 0.333$ & - & - \\
\hline & MW & $9 \pm 1.154$ & $10 \pm 0.959$ & $9 \pm 0.986$ & $8 \pm 1.000$ & - & $14 \pm 0.656$ \\
\hline & $\mathrm{MeOH}$ & $9 \pm 1.333$ & - & - & $8 \pm 0.000$ & - & $7 \pm 0.000$ \\
\hline B. bifurcata & $\mathrm{MeOH} / \mathrm{DC}$ & $7 \pm 0.881$ & - & $8 \pm 0.493$ & - & $7 \pm 0.577$ & - \\
\hline & DC & - & $7 \pm 0.000$ & - & $8 \pm 1.422$ & $10 \pm 0.577$ & - \\
\hline & EA & - & $10 \pm 0.416$ & - & $9 \pm 0.982$ & $8 \pm 0.000$ & - \\
\hline Ofloxacine $50 \mu \mathrm{g}$ & & $21 \pm 0.577$ & $30 \pm 0.907$ & $30 \pm 1.013$ & $28 \pm 0.953$ & $25 \pm 0.577$ & $30 \pm 0.866$ \\
\hline Streptomycin $100 \mu \mathrm{g}$ & & $20 \pm 0.577$ & $29 \pm 0.976$ & $23 \pm 0.768$ & $27 \pm 0.606$ & $10 \pm 0.577$ & $11 \pm 1.000$ \\
\hline
\end{tabular}

MW: methanol water (40/60), M: Methanol, M/DC: Methanol/Dichloromethane, DC: Dichloromethane (50/50), EA: Ethyl acetate,-: Resistant. Values are mean \pm standard deviation of three replicates 
The presence of a positive activity on Gram-positive and Gramnegative bacteria was observed in all seven brown algae tested (table 1). This result is similar to the one described by Ara [24] who revealed that extracts of brown algae were active against a number of Gram-positive and Gram-negative bacteria.

For Gram-positive bacteria, higher activity (was obtained against Staphylococcus aureus with methanol/water extracts of F. spiralis, $S$. muticum, C. tamariscifolia, $S$. vulgare and with dichloromethanolic extract of $S$. muticum. Against Staphylococcus epidermidis, the best inhibition was obtained with methanol/water extract of $F$. spiralis, $C$. tamariscifolia, L. ochroleuca, ethyl acetate extract of $C$. tamariscifolia, C. humilis and methanol extract of F. spiralis. For these species, others extract prepared exhibited a moderate activity against Grampositive bacteria; their diameter of the inhibition was ranged from $10 \mathrm{~mm}$ to $15 \mathrm{~mm}$.

Against Pseudomonas aeruginosa (Gram-negative bacteria), higher activity was obtained with methanol/water extracts of $F$. spiralis, $C$. tamariscifolia and $S$. vulgare. While, for Escherichia coli, the moderate activity was obtained by methanolic extract of $S$. muticum, dichloromethane/methanol extract of $F$. spiralis and with methanol/water extract of $B$. bifurcata who exhibited an important antibacterial activity against others pathogenic bacterial strains [25, $26,7]$. In earlier work, metabolite with antibacterial activity against Escherichia coli has been isolated from C. tamariscifolia and was characterized as methoxybifurcarenone [27].

Among the seven algae tested, F. spiralis exhibited higher antibacterial activity against four bacteria, followed by $C$. tamariscifolia which exhibited in important antibacterial activity against three bacteria. The present study reveals that methanol/water mixture is better for extraction of antibacterial fraction compared with other solvents. Some studies concerning the effectiveness of solvent used for extraction reported that methanol extraction yielded higher antibacterial activity than other organic solvents [28-30].
The difference between different results may be due to the strain sensitivity, seasonal variation [31-34], ecological parameters such as: temperature, salinity, light, dissolved oxygen and nutrients, or related to the biology and physiology of the seaweed itself [35] and efficiency of extraction methods to recover active metabolites. Different solvents used show different antimicrobial activity depending upon their solubility and polarity [9, 36, 37]. Therefore, chemical compounds should be extracted from different seaweeds in order to optimize their antibacterial activity by selecting the best solvent system [2].

In addition, the seven brown algae tested strong activity against Grampositive bacteria than Gram-negative bacteria which was observed. This result is in agreement with earlier reports [30, 38, 39] who proved that Gram-positive bacteria was more sensitive than Gram negative bacteria. Among six bacterial strains tested, Staphylococcus epidermidis is the most sensitive. While, Streptococcus pyogenes and Escherichia Coli are the most resistant.

The present screening revealed that the highest antibacterial activity in some brown algae indicates the presence of active compounds which can be exploited for the production of innovation drugs. In other species such as $C$. humilis, the inhibitory activity was only observed in the extract obtained with one kind of solvent but not in extracts obtained with other solvents. Which may suggest that a particular solvent is required to extract some antibacterial substances within the algal plant. Therefore, the percentage of inhibitory activity will go up when several solvents are used in the screening [2].

\section{Antifungal activity}

The results of the antifungal test of each extracts (methanol/water (60/40), methanol, dichloromethane/methanol (50/50), dichloromethane and ethyl acetate) against Candida tropicalis and Aspergillus niger are summarized in table 2.

Table 2: Antifungal activity of seven brown seaweeds extracts against Candida tropicalis and Aspergillus niger

\begin{tabular}{|c|c|c|c|c|c|c|c|c|c|c|c|c|}
\hline \multirow{3}{*}{$\begin{array}{l}\text { Seaweed } \\
\text { species }\end{array}$} & \multicolumn{12}{|c|}{ Diameter of inhibition (mm) against Candidatropicalis and Aspergillus niger } \\
\hline & \multicolumn{2}{|c|}{ MW } & \multicolumn{2}{|c|}{ MeOH } & \multicolumn{2}{|c|}{$\mathrm{MeOH} / \mathrm{DC}$} & \multicolumn{2}{|c|}{ DC } & \multicolumn{2}{|l|}{ EA } & \multicolumn{2}{|c|}{$\begin{array}{l}\text { Amphotericin B } \\
(100 \mu g)\end{array}$} \\
\hline & C. $t$ & $\begin{array}{l}A . \\
n\end{array}$ & C. $t$ & A. $n$ & C. $t$ & A. $n$ & C. $t$ & A. $n$ & C. $t$ & A. $n$ & C. $t$ & A. $n$ \\
\hline S. muticum & $\begin{array}{l}9 \pm 0.60 \\
0\end{array}$ & - & $\begin{array}{l}17 \pm 0.76 \\
3\end{array}$ & - & - & - & $\begin{array}{l}10 \pm 0.00 \\
0\end{array}$ & - & $7 \pm 0.577$ & $\begin{array}{l}12 \pm 0.50 \\
0\end{array}$ & & \\
\hline F. spiralis & $8 \pm 1.00$ & - & $\begin{array}{l}12 \pm 1.00 \\
0\end{array}$ & - & - & - & $\begin{array}{l}10 \pm 0.00 \\
0\end{array}$ & $\begin{array}{l}17 \pm 0.88 \\
1\end{array}$ & - & $\begin{array}{l}10 \pm 1.04 \\
0\end{array}$ & & \\
\hline $\begin{array}{l}\text { C. } \\
\text { tamariscifolia }\end{array}$ & $\begin{array}{l}8 \pm 1.33 \\
3\end{array}$ & - & $\begin{array}{l}10 \pm 1.52 \\
7\end{array}$ & - & - & - & - & $\begin{array}{l}14 \pm 0.50 \\
0\end{array}$ & $7 \pm 0.577$ & - & $23 \pm 1.000$ & $15 \pm 0.577$ \\
\hline S. vulgare & - & - & $9 \pm 0.731$ & - & - & - & - & $\begin{array}{l}22 \pm 0.76 \\
3\end{array}$ & - & - & & \\
\hline C. humilis & $\begin{array}{l}8 \pm 0.57 \\
7\end{array}$ & - & - & - & - & - & $7 \pm 0.000$ & - & - & - & & \\
\hline L. digitata & - & - & $\begin{array}{l}18 \pm 0.86 \\
6\end{array}$ & $\begin{array}{l}16 \pm 0.76 \\
3\end{array}$ & $\begin{array}{l}21 \pm 0.88 \\
1\end{array}$ & - & - & - & $\begin{array}{l}12 \pm 1.73 \\
2\end{array}$ & - & & \\
\hline B. bifurcata & - & - & $\begin{array}{l}18 \pm 0.57 \\
7\end{array}$ & - & $9 \pm 1.201$ & $\begin{array}{l}12 \pm 0.33 \\
3\end{array}$ & - & $7 \pm 1.000$ & - & - & & \\
\hline
\end{tabular}

C. t: Candidas tropicalis, A. n: Aspergillus Niger, MW: methanol water (50/50), M: Methanol, M/DC: Methanol/Dichloromethane (50/50), DC: Dichloromethane, EA: Ethyl acetate,-: No activity. Values are mean \pm standard deviation of three replicates

Of the seven brown algae tested, six species showed a positive activity against Candida tropicalis and Aspergillu sniger. Concerning Candidas tropicalis, very important activity was observed in the dichloromethane/methanolic extract and in methanolic extract of $L$. ochroleuca, methanolic extract of B. Bifurcata and methanolic extract of $S$. muticum (table 2). These results are in affirmation with those obtained by Rizzo [40] which shows that B. bifurcata, F. spiralis and C. Humilis possessan important antifungal activity.

Concerning, Aspergillus niger, a very important activity has been observed in the dichloromethanolic extract of $S$. Vulgare, this activity was better compared to that obtained with the control
(Amphotericin B at100 $\mu \mathrm{g}$ ): diameter of inhibition of $S$. vulgare and the control was respectively $22 \mathrm{~mm}$ and $15 \mathrm{~mm}$. Very important activity has been also observed in the dichloromethanolic extract of F. spiralisand in methanolic extract of L. ochroleuca. Wagih [41] and Saleh [3] showed that extracts of some brown algae are the most active against Aspergillus niger.

\section{Estimated minimum inhibitory concentration (MIC)}

In the current investigation MIC values, as useful parameters, have been estimated in order to screen algal inhibitory effects (table 3 ). MIC results for the algal species tested against the Different microorganisms are presented in the tables 3 and 4 . 
The extracts prepared by methanol/water solvent from $C$. tamariscifolia were more active against Gram-positive bacteria Staphylococcus aureus (MIC $=2 \mu \mathrm{g} / \mathrm{ml}$ ). Followed by methanol/water extract of $S$. muticum, $F$. spiralis and $S$. vulgare against the same strain $(\mathrm{MIC}=5 \mu \mathrm{g} / \mathrm{ml})$. The methanol/water and methanolic extracts of $F$. spiralis were more active against respectively Gram-negative bacteria Pseudomonas aeruginosa and Klepsiella pneumoniae (MIC $=5 \mu \mathrm{g} / \mathrm{ml}$ ). Followed by methanol/water extract of B. bifurcata against Escherichia Coli (MIC=10 $\mu \mathrm{g} / \mathrm{ml})$. Grozdanic [43] reported that the dichloromethane/Methanolic extract of $C$. humilis was more effective against following bacterial species: Staphylococcus aureus, Escherichia coli (MIC $=6250 \mu \mathrm{g} / \mathrm{ml}$ ) and Klebsiella pneumoniae $(\mathrm{MIC}=12500 \mu \mathrm{g} / \mathrm{ml}$ ).
Sónia and al. [44] studied brown seaweed B. bifurcata. They found that activity against Staphylococcus aureus (MIC $=1024 \mu \mathrm{g} \mathrm{ml}^{-1}$ ) and Escherichia Coli (MIC $=2048 \mu \mathrm{g} \mathrm{ml}^{-1}$ ) was observed. While no growth inhibition of Staphylococcus epidermidis and Pseudomonas aeruginosa was verified in the range of concentrations tested (MIC>2048 $\mu \mathrm{g} . \mathrm{ml}^{-1}$ ).

Our results show that $B$. bifurcate extract exhibited inhibition against both Gram-negative and Gram-positive bacteria. In opposition to that observed by Alves [45], which only verified activity of $B$. bifurcate dichloromethanolic extract against Gramnegative bacteria.

Table 3: Algal minimum inhibitory concentration (MIC) values using different solvents against pathogenic bacteria

\begin{tabular}{|c|c|c|c|c|c|c|c|}
\hline \multirow[b]{3}{*}{ Algae } & \multirow[b]{3}{*}{$\begin{array}{l}\text { Solvent } \\
\text { extraction }\end{array}$} & \multicolumn{6}{|c|}{ Minimum inhibitory concentration (MIC) $(\mu \mathrm{g} / \mathrm{ml})$} \\
\hline & & \multicolumn{3}{|c|}{ Gram+bacteria } & \multicolumn{3}{|l|}{ Gram-bacteria } \\
\hline & & $\begin{array}{l}\text { Streptococcus } \\
\text { pyogenes }\end{array}$ & $\begin{array}{l}\text { Staphylococcu } \\
\text { s aureus }\end{array}$ & $\begin{array}{l}\text { Staphylococcus } \\
\text { epidermidis }\end{array}$ & $\begin{array}{l}\text { Klepsiella } \\
\text { pneumoniae }\end{array}$ & $\begin{array}{l}\text { Pseudomonas } \\
\text { aeruginosa }\end{array}$ & $\begin{array}{l}\text { Escherichi } \\
\text { a Coli }\end{array}$ \\
\hline \multirow{5}{*}{ S. muticum } & MW & 78 & 5 & 39 & 78 & 20 & 1250 \\
\hline & M & 59 & 117 & 29 & 117 & 937 & 15 \\
\hline & $\mathrm{M} / \mathrm{DC}$ & 312 & 625 & 78 & 1250 & 156 & 2500 \\
\hline & DC & 625 & 20 & 1250 & 39 & 78 & 156 \\
\hline & EA & 2500 & 39 & 20 & 156 & 39 & 5000 \\
\hline \multirow{5}{*}{ F. spiralis } & MW & 78 & 5 & 10 & 5000 & 5 & 2500 \\
\hline & M & 15 & 59 & 29 & 7 & 469 & 117 \\
\hline & $\mathrm{M} / \mathrm{DC}$ & 78 & 312 & 39 & 625 & 78 & 20 \\
\hline & DC & 5000 & 78 & 625 & 5000 & 19 & 312 \\
\hline & EA & 1250 & 20 & 78 & 312 & 39 & 5000 \\
\hline \multirow{5}{*}{ C. tamariscifolia } & MW & 39 & 2 & 20 & 156 & 19 & 312 \\
\hline & M & 117 & 59 & 59 & 117 & 29 & 29 \\
\hline & $\mathrm{M} / \mathrm{DC}$ & 156 & 2500 & 156 & 312 & 625 & 312 \\
\hline & DC & 156 & 39 & 156 & 1250 & 625 & 1250 \\
\hline & EA & 20 & 19 & 5 & 312 & 156 & 625 \\
\hline \multirow{5}{*}{ S. vulgare } & MW & 156 & 5 & 5000 & 156 & 10 & 312 \\
\hline & M & 117 & 59 & 29 & 59 & 117 & 117 \\
\hline & $\mathrm{M} / \mathrm{DC}$ & 625 & 312 & 39 & 5000 & 78 & 1250 \\
\hline & DC & 20 & 78 & 625 & 1250 & 312 & 312 \\
\hline & EA & 20 & 19 & 78 & 39 & 1250 & 625 \\
\hline \multirow{5}{*}{ C. humilis } & MW & 5000 & 78 & 156 & 156 & 625 & 156 \\
\hline & M & 117 & 59 & 117 & 59 & 59 & 234 \\
\hline & $\mathrm{M} / \mathrm{DC}$ & 2500 & 312 & 1250 & 78 & 1250 & 625 \\
\hline & DC & 78 & 39 & 5000 & 5000 & 312 & 39 \\
\hline & EA & 39 & 78 & 39 & 78 & 156 & 39 \\
\hline \multirow{5}{*}{ L. ochroleuca } & MW & 156 & 78 & 39 & 156 & 2500 & 78 \\
\hline & M & 117 & 117 & 117 & 234 & 58 & 59 \\
\hline & $\mathrm{M} / \mathrm{DC}$ & 2500 & 625 & 625 & 78 & 78 & 2500 \\
\hline & DC & 39 & 312 & 312 & 5000 & 19 & 625 \\
\hline & EA & 312 & 20 & 156 & 78 & 1250 & 312 \\
\hline \multirow{5}{*}{ B. bifurcata } & MW & 78 & 39 & 78 & 78 & 312 & 10 \\
\hline & M & 58 & 239 & 875 & 58 & 117 & 29 \\
\hline & M/DC & 78 & 625 & 78 & 1250 & 78 & 2500 \\
\hline & DC & 312 & 156 & 5000 & 78 & 78 & 312 \\
\hline & EA & 625 & 19 & 2500 & 78 & 78 & 2500 \\
\hline Ofloxacine $50 \mu \mathrm{g}$ & & 0.97 & 0.06 & 0.06 & 0.24 & 0.97 & 0.12 \\
\hline Streptomycin $100 \mu \mathrm{g}$ & & 1.9 & 0.12 & 0.48 & 0.78 & 2.4 & 1.9 \\
\hline
\end{tabular}

MW: methanol water (40/60), M: Methanol, M/DC: Methanol/Dichloromethane, DC: Dichloromethane (50/50), EA: Ethyl acetate.

Table 4: Minimum inhibitory concentration $(\mu \mathrm{g} / \mathrm{ml})$ seaweed extracts against species of fungi

\begin{tabular}{|c|c|c|c|c|c|c|c|c|c|c|c|c|}
\hline \multirow[t]{3}{*}{ Seaweed species } & \multicolumn{12}{|c|}{ Minimum inhibitory concentration (MIC) $(\mu \mathrm{g} / \mathrm{ml})$} \\
\hline & \multicolumn{2}{|c|}{ MW } & \multicolumn{2}{|c|}{ M } & \multicolumn{2}{|c|}{ M/DC } & \multicolumn{2}{|l|}{ DC } & \multicolumn{2}{|l|}{ EA } & \multicolumn{2}{|c|}{ 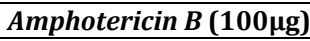 } \\
\hline & C. $t$ & A. $n$ & C. $t$ & A. $n$ & C. $t$ & A. $n$ & C. $t$ & A. $n$ & C. $t$ & A. $n$ & C. $t$ & A. $n$ \\
\hline S. muticum & 78 & 1250 & 7 & 469 & 2500 & 3750 & 78 & 312 & 156 & 78 & & \\
\hline F. spiralis & 156 & 625 & 59 & 468 & 156 & 938 & 156 & 15 & 625 & 78 & & \\
\hline C. tamariscifolia & 156 & 2500 & 117 & 234 & 2500 & 1250 & 625 & 78 & 156 & 2500 & 0.61 & 0.48 \\
\hline S. vulgare & 2500 & 312 & 117 & 234 & 2500 & 312 & 1250 & 5 & 312 & 625 & & \\
\hline C. humilis & 78 & 5000 & 938 & 1875 & 1250 & 234 & 156 & 2500 & 625 & 1250 & & \\
\hline L. ochroleuca & 625 & 5000 & 7 & 14 & 5 & 2500 & 2500 & 2500 & 39 & 5000 & & \\
\hline B. bifurcata & 625 & 2500 & 10 & 234 & 156 & 78 & 312 & 78 & 1250 & 2500 & & \\
\hline
\end{tabular}

C. $t$ : Candidas tropicalis, A. n: Aspergillus Niger, MW: methanol water (50/50), M: Methanol, M/DC: Methanol/Dichloromethane (50/50), DC: Dichloromethane, EA: Ethyl acetate,-: No activity. 
This difference in results may be due to the fact that the extraction conditions in both studies were not the same. The macroalgae origins are distinct, which may influence the metabolite composition and therefore their bioactivities. The methanol/water extract of the same seaweed exhibited the maximum growth inhibition against Staphylococcus aureus, Staphylococcus epidermidis, Pseudomonas aeruginosa and Escherichia coli (MICs were ranged between 2 to 10 $\mu \mathrm{g} / \mathrm{ml}$ ) (table 3).

The minimum inhibitory concentration was determined for $L$. ochroleuca $(\mathrm{MIC}=5 \mu \mathrm{g} / \mathrm{ml}), S$. muticum $(\mathrm{MIC}=7 \mu \mathrm{g} / \mathrm{ml}$ ) and $B$. bifurcata (MIC $=10 \mu \mathrm{g} / \mathrm{ml}$ ) as the most active extracts against Candida tropicalis. Against Aspergilis niger, the minimum inhibitory concentration was determined for $S$. vulgare (MIC $=5 \mu \mathrm{g} / \mathrm{ml}$ ), $L$. ochroleuca $(\mathrm{MIC}=14 \mu \mathrm{g} / \mathrm{ml})$ and F. spiralis $(\mathrm{MIC}=15 \mu \mathrm{g} / \mathrm{ml})$ as the most active extracts (table 4).

Saleh [46] found that the methanolic extracts of $S$. vulgare recorded a MIC of $130 \mu \mathrm{g} / \mathrm{ml}$ against Candida albicans and a MIC of $110 \mu \mathrm{g} / \mathrm{ml}$ against Aspergillus niger. However, our results show that the MIC of the methanolic extract of the same algae against Aspergilus niger is better $(\mathrm{MIC}=9.7 \mu \mathrm{g} / \mathrm{ml})$.

\section{CONCLUSION}

The seaweed extracts of seven species studied possessed noticeable activity antibacterial and antifungal against bacteria and fungi strains compared with standards solution (Ofloxacine, Streptomycin and Amphotericin B). The methanol water extracts of Cystoseira tamariscifolia, Fucus spiralis, Sargassum muticum and the dichloromethanolic extract of Sargassum vulgare, methanolic extract of Bifurcaria bifurcata will be utilized as source for extraction of antimicrobial agents. Therefore, further works may be performed on the isolation and identification of the antimicrobial components.

\section{FUNDING}

Nil

\section{AUTHORS CONTRIBUTIONS}

Both authors have contributed equally.

\section{CONFLICT OF INTERESTS}

\section{Declared none}

\section{REFERENCES}

1. Yong Xin L, Isuru W, Yong L, Se Kwon K. Phlorotannins as bioactive agents from brown algae. Process Biochem 2011;46:2219-24.

2. Kausalya GM, Narasimha Rao GM. Antimicrobial activity of marine algae. J Algal Biomass Util 2015;6:78-87.

3. Saleh B, Al-Mariri A. Antimicrobial activity of the marine algal extracts against selected pathogens. J Agric Sci Tech 2017;19:1067-77.

4. Boujaber N, Oumaskour K, Etahiri S, Assobhei O. Cytotoxic activity of some marine algae collected from the coast of sidi bouzid (El Jadida-Morocco). Int J Adv Pharm Res 2013;4:25427.

5. Mayer U, Nischt R, Poschl E, Mann K, Fukuda K, Geri M, et al. A single EGF-like motif of laminin is responsible for high affinity nidogen binding. EMBO J 1993;12:1879-85.

6. Bultel Ponce V, Etahiri S, Guyot M. New ketosteroids from the red alga Hypnea musciformis. Bioorg Med Chem Lett 2002;12:1715-8.

7. Farid Y, Chennaoui M, Assobhei 0, Etahiri S. Screening des algues marines d'Oualidia a la recherche d'activités antimicrobiennes et anti-inflammatoire. Rev Microbiol Ind San Environ 2012;6:192-209.

8. Oumaskour K, Boujaber N, Assobhei O, Etahiri S. Antiphospholipase A2 and anti elastase Activities of sixteen marine green and brown algae from the the coast of el jadida morocco. J Chem Biol Phys Sci 2015;6:101-8.

9. Tuney I, Hilal Cadirci B, Unal D, Sukatar A. Antimicrobial activities of the extracts of marine algae from the coast of Urla (Izmir, Turkey). Turk J Biol 2006;30:171-5.
10. Karabay Yavasoglu NU, Sukatar A, Ozdemir G, Horzum Z. Antimicrobial activity of volatile components and various extracts of the red alga Janiarubens. Phytother Res 2007;21:153-6.

11. Salvadora P, Begona Arti N, Querolb X, Alastueyb A, Costoyac M. Characterisation of local and external contributions of atmospheric particulate matter at a background coastal site. Atmos Environ 2007;41:1-17.

12. Boujaber N, Oumaskour K, Assobhei O, Etahiri S. Preparation of an antifungal soap from Gelidium sesquipedale waste. Afr Biodivers Conserv 2014;44:9-1.

13. Lakhdar F, Boujaber N, Oumaskour K, Assbhei O, Etahiri S. Inhibitive activity of 17 marine algae from the coast of El Jadida-Morocco against Erwinia chrysanthemi. Int J Pharm Pharm Sci 2015;7:376-80.

14. Mayer AMS. Marine pharmacology in 2003-4: marine compound with anthelmintic, antibacterial, anticoagulant, antifungal, anti-inflammatory, antimalarial, antiplatelet, antiprotozoal, antituberculosis and antiviral activities; affecting the cardiovascular, immune and nervous systems, and other miscellaneous mechanisms of action. Comp Biochem Phys 2007;145:553-81.

15. Samri N, Hsaine L, El Kafhi S, Khlifi S, Etahiri S. Radical scavenging activity and phenolic contents of brown seaweeds harvested from the coast of sidi bouzid (El Jadida, Morocco). Int J Pharm Sci Rev Res 2019;54:116-22.

16. Hsaine L, Samri N, Elkafhi S, Etahiri S, Khlifi S. Phenolic compounds and radical scavenging activity of red seaweeds harvested from the atlantic coast of sidi bouzid morocco. Int J Pharm Sci Rev Res 2019;56:73-81.

17. Hamouche E, Sarkis DK. Evolution of susceptibility to antibiotics of Escherichia coli, Klebsiella pneumoniae, Pseudomonas aeruginosa and Acinetobacter baumanii, in a University Hospital Center of Beirut between 2005 and 2009. Pathol Biol 2012;60:15-20.

18. Etahiri S, Bultel Ponce V, Caux C, Guyot M. New bromoditerpenes from the red alga Sphaerococcus coronopifolius. J Nat Prod 2001;64:1024-7.

19. Etahiri S, El Kouri A, Bultel Ponce V, Guyot M, Assobhei O. Antibacterial bromophenol from the marine red alga Pterosiphonia complanata. Nat Prod Com 2007;2:749-52.

20. Caccamese S, Azzolina R. Screening for antimicrobial activities in marine algae from Eastern Sicily. Med Plant Nat Prod Res 1979;37:333-9.

21. Bauer AW. Antibiotic susceptibility testing by a standardized single disk method. Am J Clin Pathol 1966;45:493-6.

22. Del Val AG, Platas G, Basilio A, Cabello A, Gorro Chateui J, Suay I, et al. Screening of antimicrobial activities in red, green and brown macroalgae from gran canaria (Canary Islands, Spain). Int Microbiol 2001;4:35-40.

23. Sarker SD, Nahar L, Kumarasamy Y. Microtitre plate-based antibacterial assay incorporating resazurin as an indicator of cell growth, and its application in the in vitro antibacterial screening of phytochemicals. Methods 2007;42:321-4.

24. Ara J, Sultana V, Ehteshamul Haqye S, Athar M, Qasim R. Antibacterial activity of marine macro-algae from Karachi coast. B Pol Acad Sci 2002;50:199-206.

25. Etahiri S, Butel Ponce V, EL kouri AK, Assobhei O, Zaoui D, Guyot M. Antibacterial activity of marine algae from the atlantic coast of Morocco. Mar Life 2003;13:3-9.

26. Chiheb I, Riadi H, Martine Lopez J, Dominguez Seglar JF, Gomez Vidal JA, Bouziane $\mathrm{H}$, et al. Screening of antibacterial activity in marine green and brown macroalgae from the coast of Morocco. Int Afr J Biotech 2009;8:1258-562.

27. Bennamara A, Abourriche A, Berrada M, Charrouf M, Chaib N, Boudouma M, Garneau FX. Methoxybifurcarenone: an antifungal and antibacterial meroditerpenoid from the brown alga Cystoseiratamariscifolia. Phytochemistry 1999;52:37-40.

28. Sastry A, Rao P. Antibacterial substances from marine algae: successive extraction using Benzene, chloroform and methanol. Bot Mar 1994;37:335-60.

29. Cox S, Abu Ghannam N, Gupta S. An assessment of the antioxidant and antimicrobial activity of six species of edible Irish seaweeds. Int Food Res J 2010;17:205-20. 
30. Rani V, Jawahar P, Jeya R, Shakila A, Srinivas AN. Antibacterial activity of some brown seaweeds of gulf of mannar, South East Coast of India. J Pharm Bio Sci 2016;4:14-21.

31. Perez RM, Avila JG, Perez S, Martinez A, Martinez G. Antimicrobial activity of some American algae. J Ethnopharmacol 1990;29:111-6.

32. Vidyavathi N, Sridhar KR. Seasonal and geographical variations in the antimicrobial activity of seaweeds from the Mangalore coast in India. Bot Mar 1991;34:279-84.

33. Zheng Yi, Chen Yi, LuHa. Screening for antibacterial and antifungal activities in some marine algae from the Fujian coast of China with three different solvents. Chin J Oceanol Limn 2001;19:327-31.

34. Farid Y, Etahiri S, Assobhei O. Activité antimicrobienne des algues marines de la lagune d'Oualidia (Maroc): criblage et optimisation de la periode de recolte. J Appl Biosci 2009;24:1543-52.

35. Hornesy IS, Hide D. The production of antimicrobial compounds by British Marine algae. IV variation of antimicrobial activity with algal generation. J Br Psychol 1985;20:21-5.

36. Patra JK, Patra AP, Mahapatra NK, Thatoi HN, Das S, Sahu RK, et al. Antimicrobial activity of organic solvent extracts of three marine macroalgae from Chilika Lake, Orissa, India. Malays J Microbiol 2009;5:128-31.

37. Alves C, Pinteus S, Simoes T, Horta A, Silva J, Tecelao C, et al. Bifurcaria bifurcata: a key macro-alga as a source of bioactive compounds and functional ingredients. Int J Food Sci Technol 2016;5:1638-46.

38. Rizzo C, Genovese G, Morabito M, Faggio C, Pagano M, Spano A, et al. Potential antibacterial activity of marine macroalgae against pathogens relevant for aquaculture and human health. J Pure Appl Microbiol 2017;11:1695-706.

39. Wagih A, El-shouny Reda M, Gaafar Gehan A, Ismail Marwa M, Elzanaty M. Seasonal variation of the antibacterial activity of some seaweeds against multi drug resistant pathogenic bacterial strain. Egypt J Exp Biol 2017;13:341-51.

40. Bouhraoua J, Lakhdar F, Mabrouki S, Benba J, Assobhei O, Etahiri S. Antifungal activity of 23 seaweeds from the coast of el jadida morocco against Fusarium culmorum and Alternria alternate. Int J Pharm Sci Rev Res 2018;51:53-60.

41. Erturk 0, Taş B. Antibacterial and antifungal effects of some marine algae. J Faculty Veterinary Med Kafkas University 2011;17:121-4.

42. Oumaskour K, Boujaber N, Etahiri S, Assobhei O. Screening of antibacterial and antifungal activities in green and brown algae from the coast of Sidi Bouzid (El Jadida, Morocco). Afr J Biotech 2012;11:16831-7.

43. Grozdanic N, Kosanic M, Etahiri S, Assobhei O, Kljajic Z, Stanojkovic T. An insight into cytotoxic and antimicrobial effects of Cystoseira humilis crude extract. Stud Mar 2011;29:21-30.

44. Sonia A, Santos O, Stephanie ID, Trindade S, Catia S, Oliveira D, et al. Lipophilic fraction of cultivated Bifurcaria bifurcata. R. ross: detailed composition and in vitro prospection of current challenging bioactive properties. Mar Drugs 2017;15:340.

45. Alves C, Pinteus S, Simoes T, Horta A, Silva J, Tecelao C, et al. Bifurcaria bifurcata: a key macro-alga as a source of bioactive compounds and functional ingredients. Int J Food Sci 2016;51:1638-46.

46. Saleh B, Al-Mariri A. Antifungal activity of crude seaweed extracts collected from latakia coast, Syria. J Fish Aquat Sci 2018;13:49-55 